04.2

\title{
Экспериментальное определение дисперсионного соотношения излучения плазмы на частотах ионного циклотронного резонанса и его гармоник в токамаке
}

\author{
(С) Л.Г. Аскинази, Г.И. Абдуллина, А.А. Белокуров, Н.А. Жубр, В.А. Корнев, С.В. Крикунов, С.В. Лебедев, \\ Д.В. Разуменко, А.С. Тукачинский
}

Физико-технический институт им. А.Ф. Иоффе РАН, Санкт-Петербург, Россия

E-mail: Leonid.askinazi@mail.ioffe.ru

Поступило в Редакцию 15 октября 2020 г.

В окончательной редакции 9 ноября 2020 г.

Принято к публикации 9 ноября 2020г.

\begin{abstract}
Проведено исследование спектральных свойств электромагнитного излучения ионно-циклотронного диапазона из плазмы токамака ТУМАН-3М в режиме инжекционного нагрева. Спектр этого излучения состоит из нескольких (до четырех) узких линий, соответствующих различным гармоникам частоты ионноциклотронного резонанса инжектированных быстрых ионов в центре плазмы. По задержкам сигналов пространственно разнесенных зондов определены волновые векторы, соответствующие отдельным спектральным линиям. Показано, что в предположении, что измеренные частоты и волновые векторы описываются общим дисперсионным соотношением, наблюдаемое излучение может быть объяснено возникновением в плазме неустойчивой быстрой магнитозвуковой волны, распространяющейся почти нормально к направлению магнитного поля.
\end{abstract}

Ключевые слова: ионно-циклотронное излучение, быстрые частицы, инжекционный нагрев плазмы.

DOI: 10.21883/PJTF.2021.05.50667.18585

Электромагнитное излучение из плазмы токамака на частотах ионного циклотронного резонанса и его гармониках наблюдается в режимах разряда, в которых происходит генерация быстрых ионов с энергиями, существенно превышающими тепловую. К таким сценариям относятся нагрев плазмы за счет инжекции атомарных пучков высокой энергии, ионно-циклотронный нагрев, а также режимы с генерацией и удержанием заряженных продуктов ядерных реакций синтеза: альфа-частиц, протонов, тритонов и др. Интерес к ионно-циклотронному излучению из плазмы термоядерных установок при нагреве ее инжекцией атомарных пучков (NBI ICE, от neutral beam injection и ion cyclotron emission) связан с возможностью использования его в диагностических целях, так как регистрируемое излучение является следствием возбуждения плазменных неустойчивостей популяцией быстрых ионов [1], возникающих при ионизации атомов пучка и ответственных в конечном счете за передачу энергии и импульса от пучка плазме. К числу возможных диагностических приложений относится, например, исследование изотопного состава популяции быстрых ионов, эффективности их удержания и др. В настоящей работе исследуется возможность определения дисперсионного соотношения на основе анализа спектров NBI ICE, регистрируемого магнитными зондами при инжекционном нагреве плазмы в токамаке ТУМАН-3М [2,3].

Как было обнаружено ранее [4], спектр NBI ICE в токамаке ТУМАН-3М состоит из линий, соответствующих частоте ионного циклотронного резонанса инжектируемых ионов и ее гармоникам (вплоть до четвертой) вблизи центра плазменного шнура [5]. При этом некоторые линии могут иметь тонкую структуру состоять из двух и более отдельных линий, часть из которых может быть объяснена наличием в энергетическом спектре инжектируемого пучка помимо основной компоненты с энергией $E_{0}$ составляющих с энергиями $E_{0} / 2, E_{0} / 3$ и др. Характерный пример спектра NBI ICE приведен на рис. 1, $a$. Для определения типа неустойчивости, ответственной за возникновение NBI ICE, необходимо установить дисперсионное соотношение, т.е. зависимость частоты колебаний от волнового вектора $\omega(\mathbf{k})$. В большинстве теоретических работ, посвященных этому вопросу (см., например, [6,7]), чаще всего в качестве такой неустойчивости принимается магнитозвуковая неустойчивость, вызванная взаимодействием надтепловых ионов с быстрой магнитозвуковой (БМЗ) волной. Такой волне соответствует дисперсионное соотношение вида $\omega=k v_{\mathrm{A}}$. БМЗ-волна может распространяться под произвольным (отличным от нуля) углом к магнитному полю и имеет фазовую и групповую скорости, равные альфвеновской $v_{\mathrm{A}}=B_{\mathrm{T}}\left(\mu_{0} n_{\mathrm{i}} m_{\mathrm{i}}\right)^{-0.5}$ (в случае однокомпонентной плазмы), где $B_{\mathrm{T}}-$ тороидальное поле, $n_{\mathrm{i}}$ и $m_{\mathrm{i}}$ - концентрация и масса ионов плазмы, $\mu_{0}$ - магнитная постоянная. В настоящей работе путем измерения фазовых сдвигов между сигналами магнитных зондов, разнесенных в тороидальном и полоидальном направлениях, проведено измерение компонент волнового вектора колебаний для каждой из частотных компонент спектра NBI ICE. 
Регистрируемые частоты, интервал фильтрации для модового анализа, получившиеся в результате анализа тороидальные и полоидальные модовые числа $n$ и $m$, удовлетворяющее им продольное волновое число $k_{\|}$в разряде 19021120

\begin{tabular}{l|c|c|c|c|c}
\hline \multicolumn{1}{c|}{ Обозначение } & $\begin{array}{c}f, \\
\mathrm{MHz}\end{array}$ & $\begin{array}{c}\text { Полоса } \\
\text { фильтрации, } \\
\text { МНz }\end{array}$ & $n$ & $m$ & $k_{\|}, \mathrm{m}^{-1}$ \\
\hline $\begin{array}{l}f_{1}-\text { фундаментальная } \\
\text { частота ИЦР дейтерия }\end{array}$ & 6.62 & $6.5-6.7$ & $(-4), 1,3,8$ & 1 & $3.8,7.5,17$ \\
$f_{2}$ - вторая гармоника & 13.28 & $13.2-13.4$ & $(-2), 3,10$ & 1 & $7.5,21$ \\
$\begin{array}{l}\text { (расщепление) } \\
f_{3} \text { - вторая гармоника }\end{array}$ & 13.48 & $13.4-13.6$ & $(-4), 3,8$ & 1 & $7.5,17$ \\
(расщепление) & 20.10 & $20.0-20.2$ & $(-7,0,5), 12$ & -6 & 11.3 \\
$f_{4}$ - третья гармоника & 26.92 & $26.8-27.0$ & $(-4,1), 8,13$ & -6 & $3.8,13.2$ \\
$f_{5}$ - четвертая гармоника &
\end{tabular}

Комплекс магнитной диагностики токамака ТУМАН-3М включает в себя массив из 16 внутрикамерных зондов, равномерно разнесенных по полоидальному обходу токамака. Зонды ориентированы так, чтобы регистрировать сигнал, вызванный возмущением полоидальной компоненты магнитного поля. С помощью двумерного пространственновременно́го фурье-преобразования можно определить величину и знак полоидального модового числа $m$ (т.е. направление полоидального вращения). Кроме того, имеется несколько зондов, установленных на одном полоидальном угле, но разнесенных в тороидальном направлении. Эти зонды используются для определения тороидального модового числа $n$. Продольное волновое число определялось как

$$
k_{\|}=(n+m / q) / R_{0}
$$

Знак „плюс“ в данном выражении в отличие от приведенного, например, в [8] определяется тем, что в токамаке ТУМАН-3М в этих экспериментах направление плазменного тока и тороидального магнитного поля противоположны. Здесь $q \sim 1-$ запас устойчивости в центре шнура (поскольку именно в этой области локализуются быстрые ионы, ответственные за генерацию NBI ICE в токамаке ТУМАН-3М [5]), $R_{0}$ - большой радиус плазмы.

Эксперименты по исследованию дисперсионного соотношения NBI ICE проводились в разрядах с инжекцией дейтериевого пучка с энергией $16-17.5 \mathrm{keV}$ и мощностью $\sim 250 \mathrm{~kW}$ в дейтериевую плазму (содержание водорода в пучке $<5 \%$ ). Одинаковый изотопный состав нагревного пучка и плазмы позволяет однозначно интерпретировать наблюдаемые сигналы как гармоники ионного циклотронного резонанса (ИЦР) дейтерия.

На рис. 1, $a$ и $b$ показаны спектр и спектрограмма сигнала магнитного зонда в интервале 64.5-65.5 ms, зарегистрированные в разряде 19021120. Обозначения частот расшифрованы в таблице. На спектре хорошо видна фундаментальная частота, вторая гармоника с расщеплением на две сублинии и четвертая гармоника;
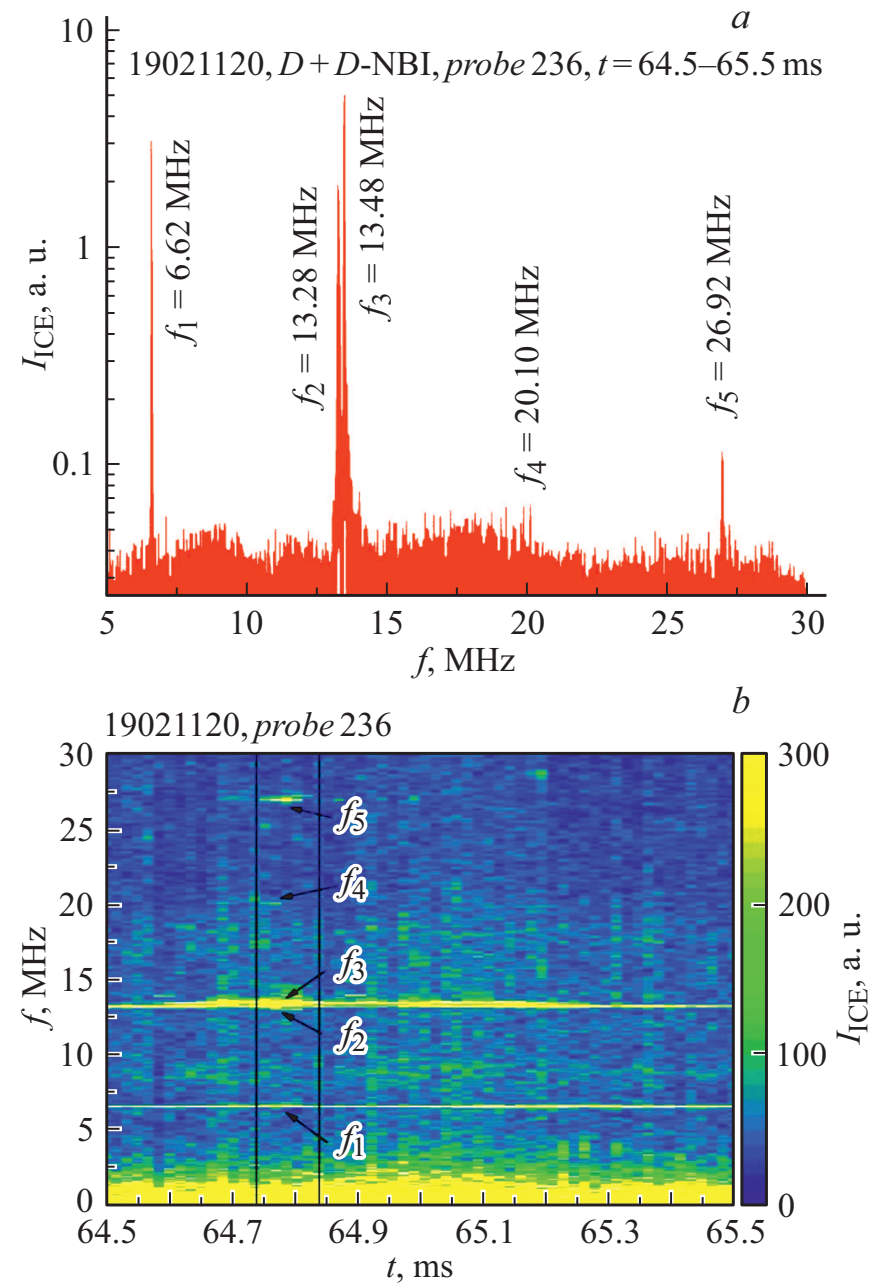

Рис. 1. Спектр $(a)$ и спектрограмма $(b)$ сигнала магнитного зонда во временно́м интервале $64.5-65.5 \mathrm{~ms}$.

третья гармоника почти не заметна на спектре, но хорошо различима на спектрограмме. Спектрограмма иллюстрирует существование фундаментальной частоты и второй гармоники достаточно продолжительное время. Анализ модового состава проводился в интервале 


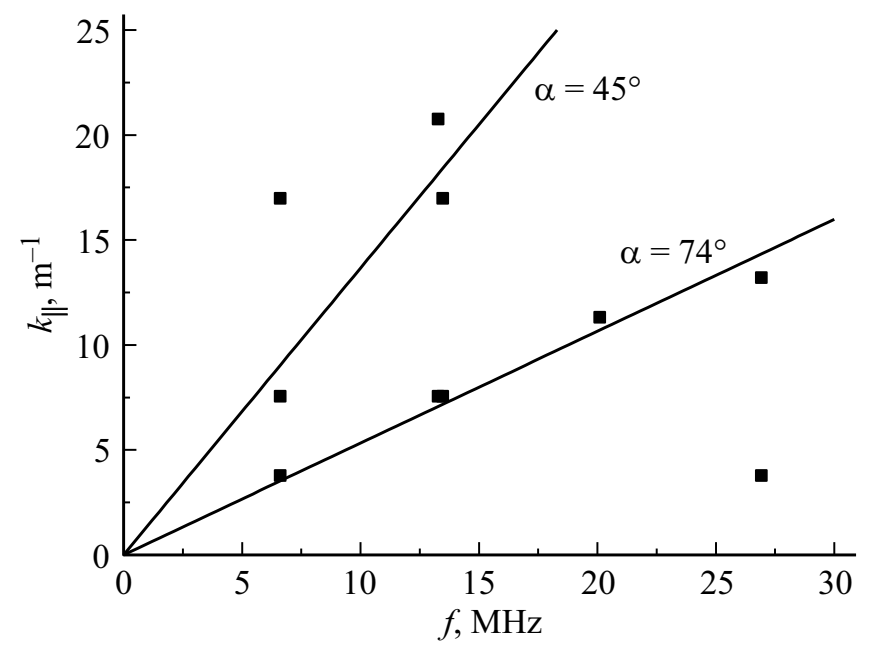

Рис. 2. Волновые числа $k_{\|}$для различных гармоник в спектре NBI ICE.

64.74-64.84 ms, обозначенном на рис. $1, b$ вертикальными линиями, в котором наблюдаются также третья и четвертая гармоники. Наличие фундаментальной частоты ИЦР дейтерия и трех ее гармоник в широком диапазоне частот от 6.62 до $26.92 \mathrm{MHz}$ позволяет установить дисперсионное соотношение для NBI ICE в предположении, что все наблюдаемые частоты соответствуют колебаниям одного типа и описываются соответственно общим дисперсионным соотношением. Для анализа модового состава каждой из линий в спектре NBI ICE сигналы магнитных зондов предварительно отфильтровывались с помощью цифровых полосовых фильтров в полосах фильтрации, указанных в таблице.

Для определения полоидального модового числа $m$ использовалось двумерное (по полоидальной координате и по времени) фурье-преобразование, позволяющее в отличие от одномерного (пространственного) преобразования определить не только модуль полоидального модового числа, но и его знак, т.е. направление вращения моды. Для анализа был выбран временной интервал в 110 периодов колебания для каждой частоты, что соответствует примерно интервалу от 4 до $16 \mu \mathrm{s}$ в диапазоне от 64.7794 до $64.7954 \mathrm{~ms}$. Для определения тороидального модового числа $n$ использовались сигналы двух магнитных зондов, разнесенных в тороидальном направлении. Выбирался временной интервал, вмещающий в себя десять периодов колебания. Он варьировался в зависимости от частоты гармоники NBI ICE. Анализировалась разность фаз сигналов зондов с учетом условия тороидальной симметрии. Следует отметить, что сдвиги фаз между сигналами двух зондов могут быть определены лишь с точностью $2 \pi N$, где $N-$ любое целое число. Для выбора $N$, т. е. для однозначного определения сдвига фаз, необходимо привлекать дополнительные соображения. Одним из таких соображений является тот факт, что волна, ответственная за возбуждение NBI ICE в токамаке ТУМАН-3М, распростра- няется навстречу инжектируемому нагревному пучку: $k_{\|} v_{\mathrm{b}}<0$ [5] $\left(v_{\mathrm{b}}-\right.$ продольная компонента скорости быстрого иона). Это условие (выполняющееся в данном разряде, хотя и не универсальное) позволяет исключить тороидальные модовые числа, выделенные в таблице скобками. На рис. 2 приведены продольные волновые числа для различных гармоник. Видно, что большинство точек группируется вблизи прямых линий, соответствующих распространению БМЗ-волны с дисперсионным соотношением $\omega=k v_{\mathrm{A}}=k_{\|} v_{\mathrm{A}} / \cos \alpha$ под углом $\alpha=45$ и $74^{\circ}$ к магнитному полю. Исходя только из имеющихся экспериментальных данных невозможно сделать обоснованный выбор между этими двумя волнами. Как известно, при скоростях инжектированных ионов ниже альфвеновской теория предсказывает почти нормальное распространение БМЗ-волны, ответственной за генерацию NBI ICE $[9,10]$, поэтому вариант волны, распространяющейся под углом $\alpha=74^{\circ}$, представляется более вероятным.

Таким образом, в работе проведено экспериментальное исследование модового состава NBI ICE, позволившее определить параметры дисперсионного соотношения неустойчивости, ответственной за его генерацию в плазме токамака ТУМАН-3М при инжекции высокоэнергичного атомарного пучка. Для измерения волнового вектора колебаний проведен анализ сдвига фаз колебаний, соответствующих частотам нескольких гармоник ионного циклотронного резонанса, регистрируемых с помощью магнитных зондов, разнесенных в тороидальном и полоидальном направлениях. В результате анализа сделан вывод, что наиболее вероятной неустойчивой волной, вызывающей NBI ICE, является БМЗ-волна, распространяющаяся под значительным углом (до $74^{\circ}$ ) к направлению магнитного поля.

\section{Финансирование работы}

Работа выполнена при поддержке Российского научного фонда (грант 16-12-10285-П „Энергичные частицы в плазме токамака“), инфраструктура токамака ТУМАН-ЗМ поддерживается за счет госзадания ФТИ им. А.Ф. Иоффе.

\section{Конфликт интересов}

Авторы заявляют, что у них нет конфликта интересов.

\section{Список литературы}

[1] K.G. McClements, R. D'Inca, R.O. Dendy, L. Carbajal, S.C. Chapman, J.W.S. Cook, R.W. Harvey, W.W. Heidbrink, S.D. Pinches, Nucl. Fusion, 55 (4), 043013 (2015). http://dx.doi.org/10.1088/0029-5515/55/4/043013

[2] L.G. Askinazi, A.G. Barsukov, V.E. Golant, V.K. Gusev, V.V. Kusnetsov, S.V. Lebedev, V.M. Leonov, A.V. Lupin, V.B. Minaev, A.A. Panasenkov, A.R. Polevoi, G.N. Tilinin, A.S. Tukachinsky, V.A. Yagnov, Plasma Devices Oper., 1 (3), 211 (2003). 
[3] Г.М. Воробьев, В.Е. Голант, С.В. Горностаев, А.В. Григорьев, М.П. Грязневич, Т.П. Евтушенко, В.А. Ипатов, А.И. Кисляков, С.В. Лебедев, Б.М. Липин, Р.Н. Литуновский, В.В. Рождественский, Н.В. Сахаров, П.П. Теплов, А.А. Федоров, К.Г. Шаховец, С.Г. Шемелинин, Физика плазмы, 9 (1), 105 (1983).

[4] Л.Г. Аскинази, Г.И. Абдуллина, А.А. Белокуров, М.Д. Блехштейн, Н.А. Жубр, В.А. Корнев, С.В. Крикунов, С.В. Лебедев, Д.В. Разуменко, А.И. Смирнов, А.С. Тукачинский, Письма в ЖТФ, 44 (22), 48 (2018). DOI: 10.21883/PJTF.2021.05.50667.18585 [Пер. версия: 10.1134/S1063785018110184].

[5] L.G. Askinazi, A.A. Belokurov, D.B. Gin, V.A. Kornev, S.V. Lebedev, A.E. Shevelev, A.S. Tukachinsky, N.A. Zhubr, Nucl. Fusion, 58 (8), 082003 (2018). https://doi.org/10.1088/1741-4326/aac10e

[6] N.N. Gorelenkov, C.Z. Cheng, E. Fredrickson, E. Belova, D. Gates, S. Kaye, G.J. Kramer, R. Nazikian, R. White, Nucl. Fusion, 42 (8), 977 (2002). https://doi.org/10.1088/0029$5515 / 42 / 8 / 306$

[7] S.E. Sharapov, M.K. Lilley, R. Akers, N. Ben Ayed, M. Cecconello, J.W.S. Cook, G. Cunningham, E. Verwichte and MAST Team, Phys. Plasmas, 21 (8), 082501 (2014). https://doi.org/10.1063/1.4891322

[8] W.W. Heidbrink, Phys. Plasmas, 15 (5), 055501 (2008). https://doi.org/10.1063/1.2838239

[9] R.O. Dendy, C.N. Lashmore-Davies, K.G. McClements, G.A. Cottrell, Phys. Plasmas, 1 (6), 1918 (1994). https://doi.org/10.1063/1.870647

[10] R. Ochoukov, K.G. McClements, R. Bilato, V. Bobkov, B. Chapman, S.C. Chapman, R.O. Dendy, M. Dreval, H. Faugel, J.-M. Noterdaeme, M. Salewski, M. Weiland, ASDEX Upgrade Team and EUROfusion MST1 Team, Nucl. Fusion, 59 (8), 086032 (2019). https://doi.org/10.1088/1741$4326 / \mathrm{ab} 2938$ 